\title{
Parameters Identification Applied to Large Space Robotic Manipulators
}

\author{
Jingting Sun \\ State Key Laboratory of Robotics and System, \\ Harbin Institute of Technology \\ Harbin, China, 18645068166 \\ sjt27@163.com
}

\author{
Shicai Shi \\ State Key Laboratory of Robotics and System, \\ Harbin Institute of Technology \\ Harbin, China
}

Fei Zhang

tate Key Laboratory of Robotics and System,

Harbin Institute of Technology Harbin, China

\author{
Xuefei Wang \\ State Key Laboratory of Robotics and \\ System, \\ Harbin Institute of Technology \\ Harbin, China
}

Hong Liu

State Key Laboratory of Robotics and System,

Harbin Institute of Technology

Harbin, China

\begin{abstract}
The dynamic parameter of the large space manipulator is the foundation of the design of the control box, the accuracy of the parameters is key to the feature of the manipulator. So a dynamic model of the flexible robotic manipulator is built, and some identification researches are carried out. Based on the model, first the off-line identification is carried out, the stiffness and the friction parameters are identified. However the manipulator work under the condition of strong radiation, high temperature switch, micro gravity space environment, some dynamic parameter can change, so a modified RLS method is introduced, with the forgetting factor taking into account, some on-line identification are carried out. All the experiments are carried out on the HIT large space manipulator and the on-line result are convergent to a stable value close to the off-line result.
\end{abstract}

Keywords-component; Manipulator joint; dynamic model; offline parameter identification; on-line parameter identification

\section{INTRODUCTION}

Large space manipulator which has the feature of low energy consumption, large ratio of load and its weight, high efficiency are used in the space station to serve and assemble, to help the orbital devices butt joint and separate, to help the astronaut do some work outside of the module ${ }^{[1]}$, even replace the astronaut to do some precise assembling work ${ }^{[2]}$, so it is the hottest research point recently. The large space manipulator is widely used in the space station, the US and Canada's MSS ${ }^{[3],[4]}$, the European's ERA ${ }^{[5]}$, for instance. The main factor that affects the dynamic accuracy and the stability is flexibility, and the flexibility of the manipulator comes from the joint and the arm ${ }^{[6]}$, because of the use of the Harmonic Drive it takes a large part of the whole flexibility. So in order to build an accurate model the parameters should be identified.

In order to fulfill the purpose, some researches are carried out on the platform of HIT large space robotic manipulator, the dynamic parameter are identified both off-line and on-line. A comparison is carried out to test the reliability of the results.

\section{HIT LARGE SPACE RoBOTIC MANIPULATOR}

As the figure 1 show, the on-ground test model of HITLarge space robotic manipulators take on the concept of module modeling to largely reduce the difference among the joints and enhance the interchangeability between different joints. The drive of the joint is permasyn motor, using the Harmonic drive to deduce the speed and transit the torque, the speed reduce-ratio is 290. There are rotary transformer and current sensor at the motor side, they are separately used to measure the position of motor and the current of the motor, at the joint side there are magnetic-encoded system and torque sensor, separately to measure the position of the joint and the quantity of the out-put torque.

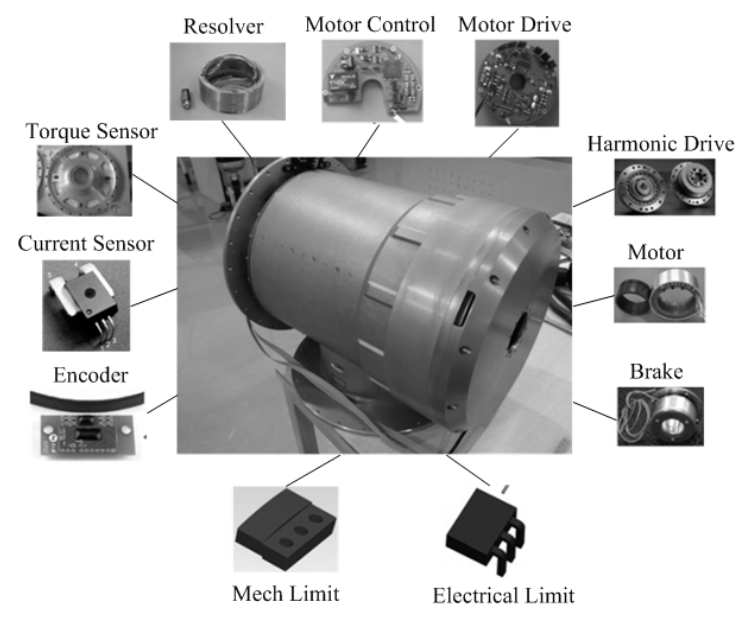

Figure 1. HIT large space robotic manipulator.

Also send a sheet of paper with complete contact information for all authors. Include full mailing addresses, telephone numbers, fax numbers, and e-mail addresses. This information will be used to send each author a complimentary 
copy of the journal in which the paper appears. In addition, designate one author as the "corresponding author." This is the author to whom proofs of the paper will be sent. Proofs are sent to the corresponding author only.

\section{DYNAMIC MODEL}

The joint model is constructed based on the following hypothesis:

Hypothesis 1: The trochanter of the motor is an ideal axisymmetric rigid body, the joint axis and the rotate-axis of the motor match together.

Hypothesis 2: View the motor as the ideal source of torque, the electric dynamics varies fast enough and ignores the influence of motor dynamics.

Hypothesis 3: Use a continuous cubic polynomial to combine the flexibility of the Harmonic Drive and the flexibility of the torque sensor together.

At present, the main methods of joint dynamic modeling are namely the Newton-Euler method [7], the Lagrange method [8] and the Kane method [9]. Among them the Newton-Euler method which has the advantage that it is in recursion form, easily calculated using numeric analysis and a clear physical meaning and high efficiency over the other methods is widely used. In this paper Newton-Euler is applied.

\section{A. Harmonic Drive Model}

The joint this paper research on use a Harmonic Drive to transmit, in order to build the dynamic model of the joint, the dynamic model of the Harmonic Drive should be built first.

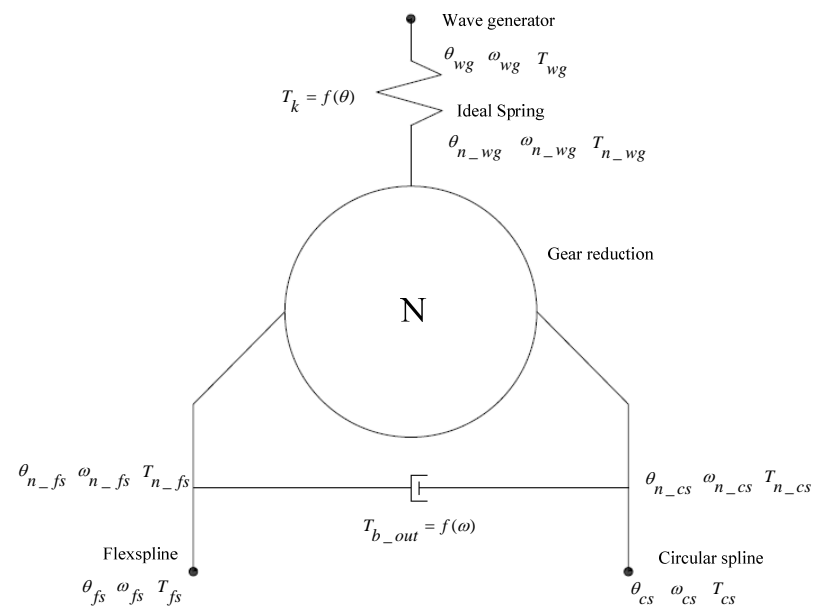

Figure 2. Simplified HD model.

In reality, during the transmission, there are some inevitable energy loss, these losses mainly come from two parts: the one at the bearing and the flexspline of the wave generator, and the one at the teeth mesh of the circular spline, among them the loss of the teeth mesh take a high proportion, so the energy loss of wave generator is ignored when modeling, regarding the energy loss of teeth mesh, and during transmission there exist large flexible deformation, therefore form the item related to the stiffness, so taking stiffness into consideration can better meet the real system model, the simplified model with regards to the energy loss and stiffness is as figure 2 shows.

In figure 2, the connections between individual torques are showed as the following equations.

$$
\begin{gathered}
T_{f s}=T_{n_{-} f s}-T_{b} . \\
T_{c s}=T_{n_{-} c s}-T_{b} . \\
T_{k}=T_{w g}=T_{n_{-} w g}=\frac{1}{(N+1)} T_{n_{-} c s}=\frac{1}{N} T_{n_{-} f s} .
\end{gathered}
$$

In order to represent the HD stiffness, several functions can be used; here we use the classical cubic model to illustrate the $T_{k}$ in Equation (3) as the Equation (4) shows.

$$
T_{k}=k_{1}\left(\theta_{w g}-\theta_{n_{-} w g}\right)+k_{2}\left(\theta_{w g}-\theta_{n_{-} w g}\right)^{3} \text {. }
$$

The HD kinematic constrains are as follows:

$$
\begin{aligned}
& \theta_{n_{-} f s}=\theta_{f s} \quad \theta_{n_{-} c s}=\theta_{c s} . \\
& \theta_{n_{-} w g}=(N+1) \theta_{c s}-N \theta_{f s} .
\end{aligned}
$$

\section{B. Dynamic Model of the Joint}

In the large space manipulator of HIT, the wave generator is attached to the motor shaft that is mounted in the circular spline. The output is obtained through flexspline. Details can be showed in figure 3 .

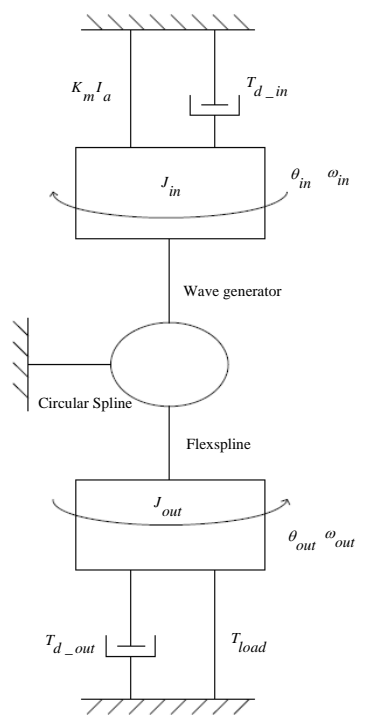

Figure 3. Simplified model of the joint. 
Based on the Newton-Euler method the dynamic model is derived, the equations show the relation between the input and the output are as follows:

$$
\begin{gathered}
J_{\text {in }} \ddot{\theta}_{\text {in }}=T_{m}-T_{d_{-} \text {in }}-T_{w g} . \\
J_{\text {out }} \ddot{\theta}_{\text {out }}=T_{\text {fs }}-T_{d_{-} \text {out }}-T_{b_{-} \text {out }}-T_{\text {load }} .
\end{gathered}
$$

Using Equation (3)(7)(8), we obtains

$$
\begin{gathered}
J_{\text {in }} \ddot{\theta}_{\text {in }}=T_{m}-T_{d_{-} \text {in }}-T_{d_{-} w g}-T_{k} . \\
J_{\text {out }} \ddot{\theta}_{\text {out }}=N T_{k}-T_{d_{-} \text {out }}-T_{b_{-} \text {out }}-T_{\text {load }} .
\end{gathered}
$$

According to the characteristic of permasyn motor, the motor torque can be considered proportional to the commanded current, thus:

$$
T_{m}=K_{m} I_{a}
$$

The damping torques $T_{d}$ on the input side, the output side, and inside the HD gear are assumed to capture two facets of damping behavior, namely a viscous and highly non-liner part $T_{\text {visc }}$, and a dry friction or Coulomb-type part $T_{\text {fric }}$ :

$$
T_{d}=T_{\text {visc }}+T_{\text {fric }} .
$$

Where, the viscous part is expected to be a liner relation in the angular velocity, as the Equation (13) shows, where $b_{1}$ is the linearly depending coefficient.

$$
T_{v i s c}=b_{1} \dot{\theta}
$$

For the dry friction part a modified classical coulomb friction model is taken, that accounts for the well-known Stribeck effects. This observes the facts that for low velocities the friction torque is normally decreasing continuously with increasing velocity, not in a discontinuous manner. What's more, with regard to the discontinuities at zero velocity, a modified model is obtained.

$$
T_{\text {fric }}=\left|T_{N}\right| \cdot\left(\mu \cdot \tanh \left(\frac{\dot{\theta}_{i}}{\omega_{1}}\right)+\frac{\dot{\theta}_{i}}{\omega_{2}} \cdot e^{-\left|\omega / \omega_{s}\right|^{\delta_{s}}}\right) .
$$

Where, $T_{N}$ is the normal torque, $\mu$ is the friction coefficient, $\omega_{s}$ is the Stribeck velocity, $\delta_{s}$ is the exponential parameter which is commonly taken either as $0.5,1$ or even 2 , here we take it as 1 .

Based on research results and experience, the frictional torque on the output side can simply cover the linearly damping and the Coulomb friction constant, while the Coulomb friction on the joint input side should use the complex model, the friction on both sides are as follows:

$$
\begin{gathered}
T_{d_{-} \text {in }}=b_{\text {in }} \dot{\theta}_{\text {in }}+C_{1} \cdot \tanh \left(\frac{\dot{\theta}_{i}}{\omega_{1}}\right)+C_{2} \cdot \dot{\theta}_{\text {in }} e^{-\left|\omega / \omega_{s}\right|^{\delta_{s}}}+b_{\text {inD }} \operatorname{sign} \dot{\theta}_{\text {in }} . \\
\text { Where, } C_{1}=\left|T_{N}\right| \cdot \mu \quad C_{2}=\frac{\left|T_{N}\right|}{\omega} . \\
T_{d_{-} \text {out }}=b_{\text {out }} \dot{\theta}_{\text {out }}+b_{\text {outC }} \operatorname{sign} \dot{\theta}_{\text {out }} .
\end{gathered}
$$

In accordance with the literature, these periodic have been introduced variations in the frictional torque captured in a sinusoidal function:

$$
T_{b_{-} \text {out }}=A_{\text {cyclic }} \sin \left(\theta_{\text {out }}+\gamma_{\text {cyclic }}\right) \text {. }
$$

As this relationship indicates, frictional torque fluctuations of amplitude $A_{\text {cyclic }}$ complete one cycle every time the flexspline makes one complete rotation relative to the circular spline. To match this model to experimental observations, a phase shift of $\lambda_{\text {cyclic }}$ is also included. In order to obtain a linear dependency of two parameters, the Equation (17) can be easily transformed to:

$$
T_{b_{-} \text {out }}=A_{1} \sin \theta_{\text {out }}+A_{2} \cos \theta_{\text {out }} .
$$

Where, $A_{1}$ and $A_{2}$ are new linearly depending parameters, they have the following equations:

$$
A_{1}=A_{\text {cyclic }} \cos \gamma_{\text {cyclic }}, A_{2}=A_{\text {cyclic }} \sin \gamma_{\text {cyclic }} \text {. }
$$

Considering the applied load on the output side, $T_{\text {load }}$ can be given:

$$
T_{\text {load }}=T_{g}
$$

During the HD transmission, the flexspline has a big deformation, so it's obvious that there exhibits a non-linear stiffness profile. Based on the information of HD manufacturer's catalog, the dependency between the applied torque and the relative angular position $\Delta \theta$ is determined by continuous piecewise functions, recent years many researchers are replacing the piecewise functions with a continuous function, it turns out that using a third order polynomial is sufficient. Here we introduce $k_{1}$ and $k_{2}$ the stiffness equation is as follows:

$$
T_{k}=k_{1} \Delta \theta+k_{2} \Delta \theta^{3} .
$$

Applying Equation (11) and Equation (21) into Equation (9) and Equation (10), we get:

$$
\begin{gathered}
J_{i n} \ddot{\theta}_{i n}-K_{m} I_{a}=-\frac{k_{1}}{N} \Delta \theta-\frac{k_{2}}{N} \Delta \theta^{3}-\tanh \left(\frac{\dot{\theta}_{\text {in }}}{\omega_{1}}\right)-\dot{\theta}_{i n} \cdot e^{-\left|\omega / \omega_{s}\right|_{s}^{s_{s}}} \\
.-b_{i n} \dot{\theta}_{i n}-b_{i n D} \operatorname{sign} \dot{\theta}_{i n}
\end{gathered}
$$




$$
\begin{aligned}
J_{\text {out }} \ddot{\theta}_{\text {out }}+T_{g} & =T_{\text {out }}=k_{1} \Delta \theta+k_{2} \Delta \theta^{3}-b_{\text {out }} \dot{\theta}_{\text {out }}-b_{\text {out }} \operatorname{sign} \dot{\theta}_{\text {out }} \\
& -A_{1} \sin \theta_{\text {out }}-A_{2} \cos \theta_{\text {out }} \cdot
\end{aligned}
$$

Where, $J_{\text {in }}$ is the input rotary inertia $\left(\mathrm{kg} \cdot \mathrm{m}^{2}\right), J_{\text {out }}$ is the output rotary inertia( $\left.\mathrm{kg} \cdot \mathrm{m}^{2}\right), K_{m}$ is the motor torque coefficient $(N \cdot m / A), I_{a}$ is the current of the motor $(A), T_{g}$ is the load torque $(N \cdot m), T_{\text {out }}$ is the output of the torque $\operatorname{sensor}(N \cdot m), \Delta \theta=\theta_{\text {out }}-\theta_{\text {in }} / N$ is the joint deformation( $\left.\mathrm{rad}\right)$, $\mathrm{N}=290$ is the speed reduction ratio, $\theta_{\text {in }}$ is the position from the input side( rad ), $\dot{\theta}_{i n}$ is the speed of the input side ( $\mathrm{rad} / \mathrm{s}$ ), $\ddot{\theta}_{i n}$ is the acceleration of the input side $\left(\mathrm{rad} / \mathrm{s}^{2}\right), \theta_{\text {out }}$ is the position from the output side $(\mathrm{rad}), \dot{\theta}_{\text {out }}$ is the speed of the output side ( $\mathrm{rad} / \mathrm{s}$ ), $\ddot{\theta}_{\text {out }}$ is the acceleration of the output side $\left(\mathrm{rad} / \mathrm{s}^{2}\right)$.

\section{JOINT PARAMETERS IDENTIFICATION PLATFORM}

The identification experiments are carried out on the platform of self-developed software and hardware in our lab. The platform mainly consists of the joint, a coder, a speed increasing gear, a torque, a magnetic powder brake. The load is added through the magnetic powder brake, the value of the torque is measured through the torque sensor. The output torque is a little too big, so the speed increasing gear is introduced to decrease the torque, figure 4 shows the platform.

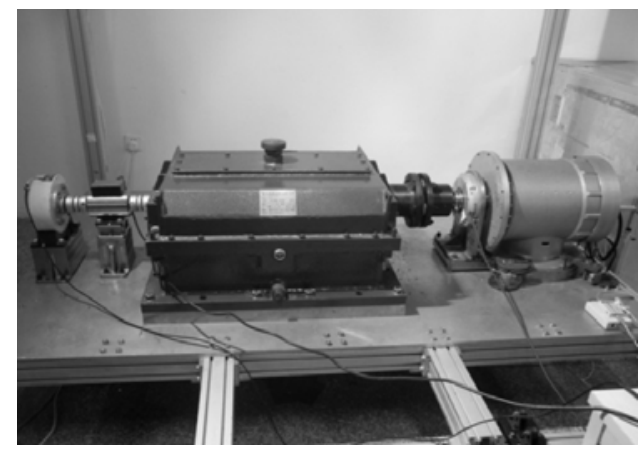

Figure 4. Parameters identification platform.

In order to make the joint to work according to a certain track under a certain load, here the classical Paul track planning is applied, the track is first accelerate, then the speed is a constant, and finally slow down; it can guarantee that during the whole course the position speed and the acceleration are continuous, and avoid the compact at the beginning and the braking stage.

\section{OFF-LINE PARAMETERS IDENTIFICATION}

Off-line parameter identification is the main way to get the engineering parameters. It using some data processing software to deal with the collecting data, and finally realize the purpose of parameters identification.
The data collected always have some singular values, so before identification so wave filtering steps should be taken to make the data more reliable. Then we carried out the identification step.

\section{A. Off-line Identification Model}

In order to make the model available for the parameters identification, the non-linear parameters are omitted, so the simplified model is as follows:

$$
\begin{aligned}
& J_{\text {in }} \ddot{\theta}_{\text {in }}=K_{m} I_{a}-\frac{k_{1}}{N} \Delta \theta-\frac{k_{2}}{N} \Delta \theta^{3}-b_{\text {in }} \dot{\theta}_{\text {in }}-b_{\text {inD }} \operatorname{sign} \dot{\theta}_{\text {in }} . \\
& J_{\text {out }} \ddot{\theta}_{\text {out }}=k_{1} \Delta \theta+k_{2} \Delta \theta^{3}-b_{\text {out }} \dot{\theta}_{\text {out }}-b_{\text {out } C} \operatorname{sign} \dot{\theta}_{\text {out }}-T_{g} .
\end{aligned}
$$

Applying Equation (24) and Equation (25) into Equation (26) and Equation (27), we obtain:

$$
\begin{gathered}
J_{\text {in }} \ddot{\theta}_{\text {in }}-K_{m} I_{a}=-\frac{k_{1}}{N} \Delta \theta-\frac{k_{1}}{N} \Delta \theta^{3}-b_{\text {in }} \dot{\theta}_{\text {in }}-b_{\text {inD }} \operatorname{sign} \dot{\theta}_{\text {in }} . \\
J_{\text {out }} \ddot{\theta}_{\text {out }}+T_{g}=k_{1} \Delta \theta+k_{2} \Delta \theta^{3}-b_{\text {out }} \dot{\theta}_{\text {out }}-b_{\text {out } C} \operatorname{sign} \dot{\theta}_{\text {out }} .
\end{gathered}
$$

From the torque sensor we obtain:

$$
T_{\text {out }}=J_{\text {out }} \ddot{\theta}_{\text {out }}+T_{g} \text {. }
$$

\section{B. Off-line Identification Experiments}

During the identification we chose the stage when the speed is constant, so the accelerate item can be avoided. The experiment can be divided into two parts: the part that considering damping torque, and the part ignoring the damping torque.

After the damping items are omitted, the model is simplified as follows:

$$
y=\left(\begin{array}{c}
y_{1} \\
y_{2}
\end{array}\right)=\left(\begin{array}{c}
-K_{m} I_{a} \\
T_{\text {out }}
\end{array}\right)=\left(\begin{array}{c}
-\frac{k_{1}}{N} \Delta \theta-\frac{k_{2}}{N} \Delta \theta^{3} \\
k_{1} \Delta \theta+k_{2} \Delta \theta^{3}
\end{array}\right) .
$$

Apparently the Equations (29) have the relationship $y_{2}=-N \cdot y_{1}$, if we treat the Equation(29) as decoupling, we will get four parameters, two for each $k_{1}$ and $k_{2}$, then considering the coupling relationship, we use $y_{2}$ minus $N \cdot y_{1}$ then we obtain:

$$
\frac{T_{\text {out }}+N \cdot K_{m} I_{a}}{2}=k_{1} \Delta \theta+k_{2} \Delta \theta^{3} .
$$

Based on the coupling and decoupling model we obtained the following results:

TABLE I. IDENTIFICATION RESULTS IGNORING THE DAMPING TORQUE

\begin{tabular}{|c|c|c|c|c|}
\hline Condition & \multicolumn{2}{|c|}{$k_{1}(\mathbf{N m} / \mathbf{r a d})$} & \multicolumn{2}{c|}{$k_{2}\left(\mathbf{N m} / \mathbf{r a d}^{3}\right)$} \\
\hline decoupling & $4.635 \times 10^{5}$ & $4.786 \times 10^{5}$ & $1.836 \times 10^{11}$ & $1.732 \times 10^{11}$ \\
\hline coupling & \multicolumn{2}{|c|}{$4.7105 \times 10^{5}$} & \multicolumn{2}{|c|}{$1.784 \times 10^{11}$} \\
\hline
\end{tabular}


In this identification all the damping torques are omitted, under this condition the stiffness coefficient is 1.2 times of the manufacturer's catalog, so the influence of the damping torque cannot be avoided.

Then take the damping torque into consideration, and first only use the simple Coulomb model, ignore the viscous damping and the periodic friction between gear teeth, so the model is simplified as:

$$
\left(\begin{array}{c}
-K_{m} I_{a} \\
T_{\text {out }}
\end{array}\right)=\left(\begin{array}{c}
-\frac{k_{1}}{N} \Delta \theta-\frac{k_{2}}{N} \Delta \theta^{3}-b_{\text {inD }} \operatorname{sign} \dot{\theta}_{\text {in }} \\
k_{1} \Delta \theta+k_{2} \Delta \theta^{3}-b_{\text {outC }} \operatorname{sign} \dot{\theta}_{\text {out }}
\end{array}\right) .
$$

Without considering the coupling relationship, the results are showed in table 2:

TABLE II. IDENTIFICATION RESULTS IGNORING THE DAMPING TORQUE

\begin{tabular}{|l|l|l|l|l|}
\hline & $k_{1}(\mathrm{Nm} / \mathrm{rad})$ & $k_{2}\left(\mathrm{Nm} / \mathrm{rad}^{3}\right)$ & $b_{\text {inD }}(\mathrm{Nm})$ & $b_{\text {outC }}(\mathrm{Nm})$ \\
\hline Eq1 & $4.325 \times 10^{5}$ & $1.536 \times 10^{11}$ & 0.2324 & - \\
\hline Eq2 & $4.486 \times 10^{5}$ & $1.639 \times 10^{11}$ & - & 83.1388 \\
\hline
\end{tabular}

Through comparison when considering the Coulomb friction the results are much closer to the manufacturer's catalog, so the Coulomb torque should not be ignored.

As we all know, when the greasing method is grease greasing or liquid greasing, there exists the viscous damping torque varies with the speed, so we now take the viscous damping into consideration, here we obtain:

$$
\left(\begin{array}{c}
-K_{m} I_{a} \\
T_{\text {out }}
\end{array}\right)=\left(\begin{array}{c}
-\frac{k_{1}}{N} \Delta \theta-\frac{k_{2}}{N} \Delta \theta^{3}-b_{\text {in }} \dot{\theta}_{\text {in }}-b_{\text {inD }} \operatorname{sign} \dot{\theta}_{\text {in }} \\
k_{1} \Delta \theta+k_{2} \Delta \theta^{3}-b_{\text {out }} \dot{\theta}_{\text {out }}-b_{\text {out } C} \operatorname{sign} \dot{\theta}_{\text {out }}
\end{array}\right) .
$$

Considering the coupling relationship of the Equation 32, we have the result as follows:

TABLE III. IDENTIFICATION RESULTS IGNORING THE DAMPING TORQUE

\begin{tabular}{|c|c|c|c|c|c|}
\hline $\begin{array}{c}k_{1} \\
(\mathrm{Nm} / \mathrm{rad})\end{array}$ & $\begin{array}{c}k_{2} \\
\left(\mathrm{Nm} / \mathrm{rad}^{3}\right)\end{array}$ & $\begin{array}{c}b_{\text {in }} \\
(\mathrm{Nms} / \mathrm{rad})\end{array}$ & $\begin{array}{c}b_{\text {inD }} \\
(\mathrm{Nm})\end{array}$ & $\begin{array}{c}b_{\text {out }} \\
(\mathrm{Nms} / \mathrm{rad})\end{array}$ & $\begin{array}{c}b_{\text {outC }} \\
(\mathrm{Nm})\end{array}$ \\
\hline $3.965 \times 10^{5}$ & $1.336 \times 10^{11}$ & 0.035 & 0.135 & 635.663 & 30.96 \\
\hline
\end{tabular}

By comparing all the three results we got with the manufacturer's catalog, we reached that by considering the Coulomb and the viscous damping torque the result can better fit the information from the manufacturer's catalog, thus can better reflect the dynamic feature of the joint, so the Coulomb and the viscous damping cannot be ignored.

\section{ON-LINE PARAMETERS IDENTIFICATION}

The on-line parameters identification is gathering data when the system is on, can through some method, the data are processed, finally the parameters vary with time can be obtained.
The on-line identification mainly have two steps, the first one is gathering data, can then processing the data, and finally through the certain method we get the parameters.

The off-line identification belong to batch processing method, its main idea is first to gather data through sensor and then use the RLS method to process the data, even though we can get the high accuracy parameter, it takes more memory and a lot of calculation, cannot get the parameters vary with time. So in this paper we use an online parameter identification based on the HIT large space manipulator, namely the modified version of RLS method.

\section{A. Modified Version of RLS}

The modified version of RLS, based on the old data and take the new data into account, after recurrence, the parameters are convergence to the expected value. It can provide a valid method to estimate the parameters, here is the principle:

By assuming a time discrete model:

$$
y(t+1)=a_{1} x_{1}(t)+\cdots+a_{n} x_{n}(t)=\varphi^{T}(t) \Theta(t) .
$$

Where, $\Theta^{T}=\left[\begin{array}{llllll}a_{1} & \cdots & a_{n} & b_{1} & \cdots & b_{n}\end{array}\right]$ is the unknown parameters vector, and $\varphi^{T}=\left[\begin{array}{llllll}x_{1} & \cdots & x_{n} & u_{1} & \cdots & u_{n}\end{array}\right]$ is the measurement vector.

An adjustable predictor model can be describe by:

$$
\hat{y}(t+1)=\varphi^{T}(t) \hat{\Theta}(t)
$$

The RLS goal is to minimize the quadratic error:

$$
J(t)=\frac{1}{N} \sum_{i=1}^{t} \frac{1}{2} \varepsilon(\Theta, i) \varepsilon(\Theta, i)^{T} .
$$

Where, $\varepsilon(\Theta, i)=y(i)-\varphi^{t}(i) \Theta$ is the error.

The optimal value of $\Theta(t)$ based on LS approach using all data available until instant $\mathrm{t}$ is:

$$
\hat{\Theta}(t)=\left[\sum_{i=1}^{t} \varphi(i-1) \varphi(i-1)^{T}\right]^{-1}\left[\sum_{i=1}^{t} \varphi(i-1) y(i)\right] .
$$

Defining a $\bar{R}(t)$ matrix and a vector $f(t)$

$$
\bar{R}(t)=\sum_{i=1}^{t} \varphi(i-1) \varphi(i-1)^{T}, f(t)=\sum_{i=1}^{t} \varphi(i-1) y(i) .
$$

Then Equation (37) can be written as:

$$
\Theta(t)=\bar{R}^{-1}(t) f(t)
$$

In the recursive form:

$$
\begin{gathered}
\bar{R}(t+1)=\lambda(t) \bar{R}(t)+\varphi(t) \varphi(t)^{T} . \\
f(t+1)=\lambda(t) f(t)+\varphi(t) y(t+1) . \\
\hat{\Theta}(t+1)=\bar{R}^{-1}(t+1) f(t+1) .
\end{gathered}
$$


Where, $\lambda(t)$ is the forgetting factor that will weigh the identification process based on the previous estimation preformation.

Applying Equation (39) and Equation (40) in Equation (41) and using Equation (38), we obtain:

$$
\hat{\Theta}(t+1)=\hat{\Theta}(t)+\bar{R}^{-1}(t+1) \varphi(t) \varepsilon(\hat{\Theta}, t) .
$$

By applying the matrix inversion lemma in Equation (42), Equation (42) can be written as (43):

$$
\hat{\Theta}(t+1)=\hat{\Theta}(t)+L(t+1) \varepsilon(\hat{\Theta}, t) .
$$

Where, $L(t+1)=\frac{P(t) \varphi(t)}{\lambda(t)+\varphi(t)^{T} P(t) \varphi(t)}, P(t)=\bar{R}(t)^{-1}$

$P(t+1)=\frac{1}{\lambda(t)}\left[P(t)-L(t+1) \varphi(t)^{T} P(t)\right]$.

\section{B. Forgetting factor}

In the modified version of RLS, the chosen of the forgetting factor is key to the convergence of the method, the quality of the identification result depends on the forgetting factor.

The forgetting factor is used to distribute the weight between the old result and the new data; it can prevent the result from diverging, and increase the convergent speed.

In this paper, a forgetting factor model based on error is presented; this model can not only track those time-varying parameters, but also those time-invariant parameters, if the system is time invariant, the forgetting factor is closed to 1 , and if the system is time variant, the factor is changing with the variation of the error.

Based on the principle above, a function is formed relates the relative error and the forgetting factor, here we chose the Gauss function:

$$
\lambda(\bar{\varepsilon})=(1-\alpha)+\alpha e^{\beta \bar{\varepsilon}^{2}}
$$

Where, $\alpha$ is the variance ratio, $\beta$ is the error coefficient, $\bar{\varepsilon}$ is the relative error.

To make the variation tendency of the relative error $\bar{\varepsilon}$ with the forgetting factor $\lambda(\bar{\varepsilon})$, here we make $\alpha=0.05, \beta=-4.68$, figure 4 shows the curve.

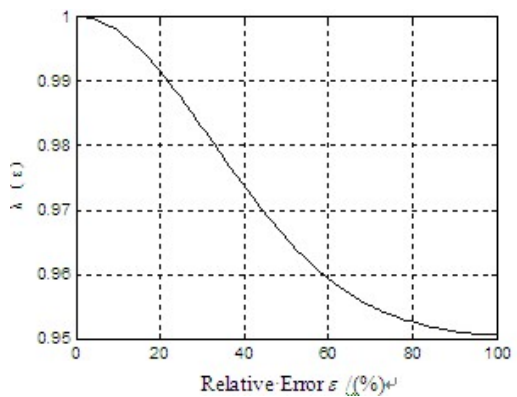

Figure 5. Forgetting factor based on relative error.
The forgetting factor model based on the relative error can make the model converge faster, so finally this model is adopted.

\section{On-line identification experiments}

The modified RLS can only be adopted to those linearly parameters identification, it cannot deal with the nonlinearly parameters identification, so those nonlinear parameters are omitted when it is applied to the large space manipulator, here we use the same model as the off-line parameters identification.

Here we use the Paul track planning to plan the track, thus guarantee the continues of the position, speed, accelerate, what's more similar to the off-line identification, we chose the middle course where the speed is constant to identify, thus can avoid the accelerate; here we have the assumption that the parameter won't vary too much during the course. So the model can be simplified as follows:

$$
\left(\begin{array}{c}
-K_{m} I_{a} \\
T_{\text {out }}
\end{array}\right)=\left(\begin{array}{c}
-\frac{k_{1}}{N} \Delta \theta-\frac{k_{2}}{N} \Delta \theta^{3}-b_{\text {in }} \dot{\theta}_{\text {in }}-b_{\text {in C }} \operatorname{sign} \dot{\theta}_{\text {in }} \\
k_{1} \Delta \theta+k_{2} \Delta \theta^{3}-b_{\text {out }} \dot{\theta}_{\text {out }}-b_{\text {out } C} \operatorname{sign} \dot{\theta}_{\text {out }}
\end{array}\right) .
$$

The modified RLS is a kind of on-line parameters identification method based on the joint dynamic model. During the course part of the old data are replaced by the new data, and finally in convergence to the ideal value, thus fulfil the parameter identification.

Some data are gathered in the stage where the speed is constant, here we use the off-line result to make the on-line identification converge fast, and then though the iteration we reached the expected value.
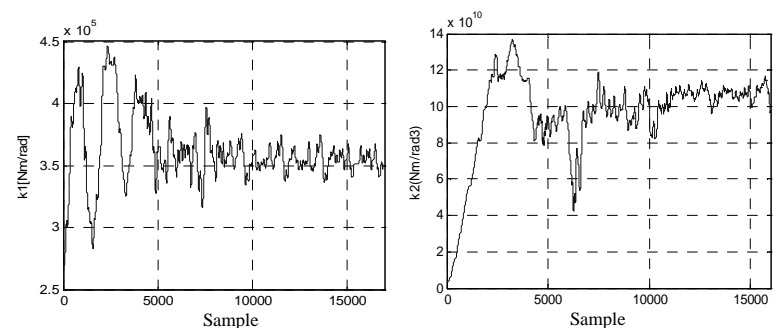

Figure 6. Stiffness coefficient $k_{1}$ and $k_{2}$.
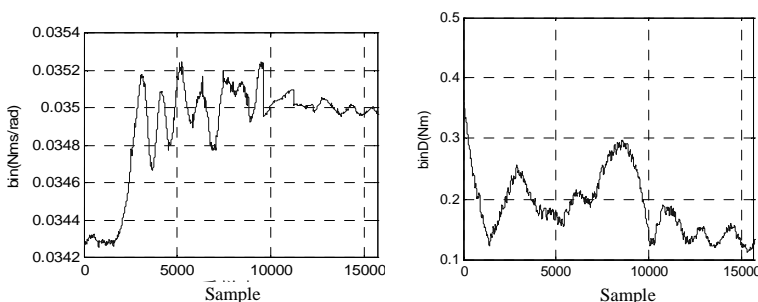

Figure 7. Input damping torque ${ }^{b_{\text {in }}}$ and ${ }^{b_{\text {inD }}}$. 

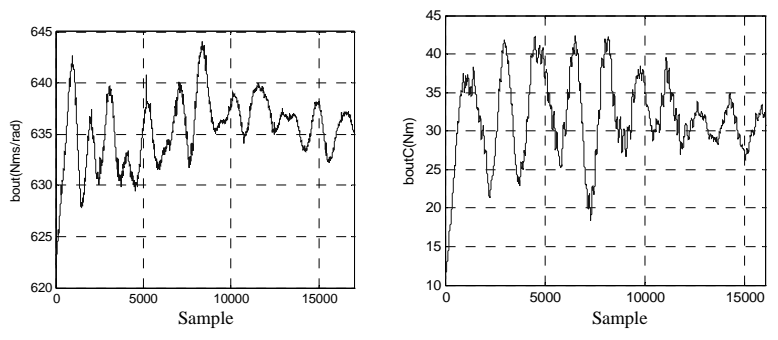

Figure 8. Output damping torque $b_{\text {out }}$ and $b_{\text {inc }}$.

By changing the forgetting factor to adjust the convergent speed, the parameter is attempted to adjust during the course.

From the figures above, it is clear that the stiffness coefficients $k_{1}$ and $k_{2}$, the input damping torque coefficients $b_{\text {in }}$ can $b_{\text {inD }}$, the output damping torque coefficients $b_{\text {out }}$ and $b_{\text {outc }}$ are all in convergence to expected values. The expected values are in accordance to the off-line parameters identification results, however, there are some fluctuation around the expected value, a little dig to the reason, in motion these parameters vary at different position or under this experiment the parameters are not well excited, what's more, the gear box has its weakness when transmitting and the magnetic powder brake also have some fluctuation.

\section{CONCLUSION}

With flexibility and friction taking into account, a dynamic model is proposed; the off-line parameters identification investigation has been carried out based on the simplified model, and the result shows that Coulomb friction and damping model can better reflect the real joint dynamic feature. The
RLS is presented, where a variable forgetting factor is introduced, then the on-line parameters identification has been investigated, and the result presents that the parameters can all reached to a stable expected value, and closed to the off-line results. The parameters identification can pave the way for joint control design.

\section{REFERENCES}

[1] G. Hirzinger, B. Brunner, "ROTEX-the first remotely controlled robot in space,” IEEE International Conference on Robotics and Automation, San Diego, USA, 1994, pp. 2604-2611.

[2] A. Albu-schaffer, W. Bertleff, B. Rebele, et al. "ROKVISS-Robotics component verification on ISS current experimental results on parameter identification,” Proceedings of the 2006 IEEE International Conference on Robotics and Automation. Orlando, Florida, USA, 2006, pp. 38803885.

[3] C. Sallaberger. "Canadian space robotic Activities," Acta Astronautica, vol.41, no.4, pp. 239-246, 1997.

[4] O. Ma, J. Wang, Misra, e tal. "On the Validation of SPDM Task Verification Facility,” Journal of Robotic Systems, vol.21, no.5, pp. 219235, 2004.

[5] http://www.esa.int/esaHS/ESAQEI0VMOC_iss_0.html

[6] Z. C. Qiu, D.L. Tan , "On acceleration sensor-based feedback control for contact force of the flexible joint manipulator," Chinese Journal of Mechanical Engineering, vol.38, no.10, pp. 37-42, 2002.

[7] V. O. Gamarra-rosado, E. A. O. Yuhara, "Dynamic Modeling and Simulation of a Flexible Robotic Manipulator,” Robotica, vol.17, no.5, pp.523-528, 1999.

[8] S. H. Hu, L. J. Xue, W. F. Xu, et al. "Dynamic Simulation System of Space Robot with Series Rotary Joint,” Journal of Jilin University (Engineering and Technology Edition), vol.38, no.2, pp.260-267, 2008.

[9] R. L. Huston, "Multi-body Dynamics including the Effects of the Flexibility and Compliance,” Computers and Machines, vol.14, no.5, 